\title{
Outage Probability of a Multi-Relay Cognitive Network with an Uncertain Number of Forwarding Relays
}

\author{
Yulin $\mathrm{Hu}^{\dagger}$, James Gross ${ }^{\dagger \dagger}$ and Anke Schmeink ${ }^{\dagger}$ \\ ${ }^{\dagger}$ RWTH Aachen University, Germany, Email: $\{h u \mid$ schmeink $\} @$ umic.rwth-aachen.de \\ ${ }^{\dagger \dagger}$ KTH Royal Institute of Technology, Sweden, Email: james.gross@ee.kth.se
}

\begin{abstract}
In this paper, we focus on a Cognitive Relay Network (CRN) where only the information of the average channel gains is available at the secondary source. A distributed multiple relay selection scheme is proposed for the secondary transmission. Since the secondary source does not fully control the selection but lets each secondary relay decide by itself, it doesn't know which and how many relays will participate in the forwarding. This brings a serious uncertainty in the number of forwarding relays. We analyze this uncertainty and derive the closed-form expression of the outage probability of the secondary transmission under the uncertainty. Finally, we evaluate the proposed relay selection scheme by means of simulation. The simulation shows the appropriateness of our analytical model. In addition, although having lower interference constraint at each relay, the performances of the proposed multi-relay CRN scheme are shown to be strictly superior to the single relay CRN. Moreover, the simulation results suggest that it is necessary to spend the general overhead for secondary networks on knowing the exact QoS requirement of the primary user (i.e. the interference violation probability) to apply our proposed relay selection scheme with the statistical total interference constraint.

Index Terms-cognitive relay networks, outage probability,
\end{abstract} relay selection, decode-and-forward, average channel gain

\section{INTRODUCTION}

Cognitive radio is emerging as a promising technology that enables secondary users (SU) to receive signals from the secondary source (SS) over licensed bands through detecting spectrum holes. In cognitive radio networks, SUs can be serviced over the spectrum of primary networks as long as the secondary transmission safeguards the quality of service of the primary users (PU) [1]. On the other hand, relaying is well known as an effective way to mitigate the wireless channel-fading by exploiting the spatial diversity gain [2], [3]. Specifically, when multiple relays are available to assist the service, the performance of the transmission can be significantly improved [4], [5]. As a result, cognitive relay networks (CRN) have been of huge interest [6]-[11].

The outage probabilities of the CRNs have been derived with considering the impact of the spectrum sensing accuracy in overlay coexistence [6], [12]. In addition, the performances of both PUs and SUs are studied in a CRN in comparison to the conventional relay networks [7]. For CRNs with multiple

978-1-4799-5863-4/14/\$31.00 (c) 2014 IEEE secondary relays (SR), only selecting best SRs for assisting the secondary transmission can significantly reduce the interference to the PU. The outage probability of the secondary transmission based on the $N$ th best-SR selection is studied in [8]. An energy efficient relay selection is proposed for CRNs based on the perfect channel state information (CSI) at SS and SU [9]. To select a single SR which is expected to have a strong link to SS but weak link to PU, [10] applies a max-min relay selection scheme for a CRN where all the instantaneous CSI are assumed to be available at the SS. Also with the instantaneous CSI at the SS, the best single SR with the highest signal-to-interference-ratio is selected for the CRN [11].

In order to select best relays for SU while safeguarding the quality of PU's service, the above existing solutions assume instantaneous CSI of all the links are available at the SS. Unfortunately, this is over-optimistic in practice, especially for a CRN with larger number of SRs. Hence the study of low-overhead-based distributed multiple relay selection will be very beneficial for the design of realistic CRN. In particular, it is still an open problem how the secondary transmissions of CRNs safeguard the quality of the PU's service while the SS does not have the instantaneous CSI.

In this paper, we consider a CRN system where the SS only has the knowledge of the average channel gains of all the links. We assume each SR has the instantaneous CSI of the link from itself to the PU but does no share the CSI with other relays or feedback it to the SS. In order to control the sum of (SR) interference to PU, we introduce a distributed multiple relay selection scheme for the CRN to guarantee the PU service. We consider two different interference constraint policies "absolute total interference constraint" (AIC) and "statistical total interference constraint" (SIC) in the proposed relay selection scheme. In the process of the proposed distributed relay selection, the SS doesn't know which and how many SRs will be selected and participate in the forwarding frame. As one contribution of this paper, we derive the closedform expression of the outage probability of the secondary transmission under this uncertainty. Furthermore, the minimal outage probability of the multi-relay CRN system is studied. We find that the minimal outage probability of a relay-assisted secondary transmission is only subject to the interference links. 
In addition, the single SR and multiple SRs assisted secondary transmissions have the same minimal outage probabilities under the AIC strategy. At last, we evaluate the relay selection scheme and show the appropriateness of our analytical model by means of simulation. We show that the SIC is superior to the AIC only in multi-relay CRNs. The results suggest these multi-relay CRNs (where only average CSI is available at the $\mathrm{SS}$ ) to spend the general overhead for secondary networks on knowing the exact interference violation probability requirement of the PU. Moreover, it is also shown that the multi-relay CRN performances are absolute superior to the single relay $\mathrm{CRN}$ while the advantage of multi-relay $\mathrm{CRN}$ is lower when the PU is getting close to the SRs.

The rest of the paper is organized as follows. Section II introduces the multi-relay CRN model. In Section III, a distributed multi-relay selection scheme is proposed. In addition, the outage probability of the multi-relay selection scheme is studied with two interference constraint policies. By means of simulations, Section IV evaluates the relay selection scheme and shows the appropriateness of our analytical model. Finally, we conclude the paper in Section V.

\section{System Model}

We consider a CRN in which a PU coexists with several secondary nodes as schematically shown in Fig. 1. Among the

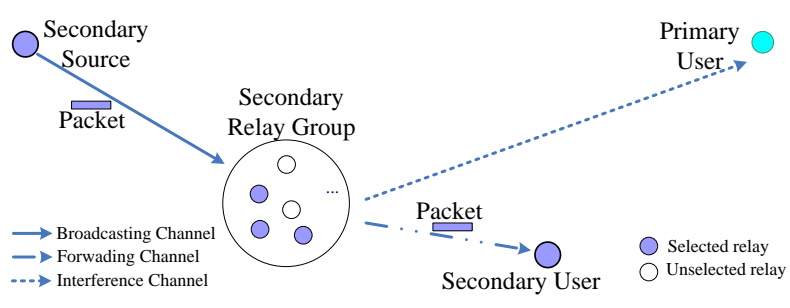

Fig. 1. A cognitive relay network

secondary nodes, a SS is required to transmit a data packet of size $\rho$ to its destination SU. We assume that the SS is far alway from both SU and PU. Therefore, the signals from the SS are very weak for both the PU and the SU. A group of secondary decode-and-forward relays are deployed to help the secondary transmission, which bring considerable interferences to the PU. The group $\left\{\mathrm{R}_{1}, \ldots \mathrm{R}_{J}\right\}$ has $J$ relays which are randomly deployed in a certain area while the radius of the area is significantly smaller than the distance either from the SS to the relay group or from the relay group to the SU or the PU.

In the secondary transmission, time is divided into frames of length $T_{\mathrm{f}}$. A single secondary transmission requires two frames on transmitting the packet from the SS to the SU, which is referred to as broadcasting frame and forwarding/relaying frame. During a broadcasting frame, the SS transmits the data packet to all SRs. And afterwards, some selected SRs forward the packet to the SU in a relaying frame. We assume that the channel states vary randomly due to a Rayleigh-distributed block-fading process. All the secondary transmitters apply fixed transmit powers denoted as $P_{\mathrm{S}}$ at the $\mathrm{SS}$ and $P_{\mathrm{R}}$ at each SR. The noise power is denoted as $\sigma^{2}$.
We assume that each relay has the instantaneous CSI of the channel from itself to the primary user while the source only has the knowledge of the average channel gains. Denote the channel gain during a broadcasting frame from the SS to $\mathrm{R}_{j}$ by $h_{\mathrm{S}, j}^{2}$. Then, the signal-to-noise-ratio (SNR) of the broadcasting frame is given as $\gamma_{\mathrm{S}, j}=P_{\mathrm{S}} \cdot h_{\mathrm{S}, j}^{2} / \sigma^{2}$. Correspondingly, the channel gain and SNR from $\mathrm{R}_{j}$ to $\mathrm{SU}$ are given by $h_{j, \mathrm{D}}^{2}$ and $\gamma_{j, \mathrm{D}}=P_{\mathrm{S}} \cdot h_{j, \mathrm{D}}^{2} / \sigma^{2}$. If the gain of the channel from a selected relay $\mathrm{R}_{j}$ to the $\mathrm{PU}$ is given by $h_{j, \mathrm{P}}^{2}$, the corresponding interference power can be obtained by $P_{\mathrm{R}} \cdot h_{j, \mathrm{P}}^{2}$. Regarding the interference constraint, which limits the total interference from all relays to PU, we consider it in the relay selection process and do not assume any power control at each relay. As the distance among the relays is fairly small compared to the distances from SRs to the SS or to the SU and PU, we make a topology simplification that all the relays have the same "average channel gains" for broadcasting channels denoted by $\bar{h}_{\mathrm{B}}^{2}$, for relaying channels denoted by $\bar{h}_{\mathrm{R}}^{2}$ and for interference channels denoted by $\bar{h}_{\mathrm{I}}^{2}$. All the three average channel gains are available at the SS.

Given an instantaneous SNR $\gamma$ during one frame (with $N$ symbols), at most $N \cdot \log _{2}(1+\gamma)$ bits can be conveyed correctly. Hence, a currently transmitted packet of size $\rho$ is successfully received if the SNR of the link is above the threshold $\gamma^{*}=2^{\rho / N}-1$.

\section{Distributed MultiPle RELAY SELECTION SCHEME}

In this section, a distributed multi-relay selection scheme is proposed. Based on it, we analyze the outage probability of the secondary transmission.

\section{A. The Process of Selection}

The distributed multiple relay selection process has two steps. A parameter plays the role of a baton in the selection process, which is called single relay interference constraint (SRIC) and denoted by $I_{\mathrm{R}}$. In the first step, the SS is required to decide the value of SRIC based on the information of the average channel gains, then the SS broadcasts the value $I_{\mathrm{R}}$ to all SRs once at the beginning of each time relay selection. After that, the source broadcasts the data packet to all the SRs. Due to the fading, a varying amount of SRs could decode the packet successfully during the broadcasting phase. We call this set the surviving relay set and denote it by $\Theta_{\mathrm{s}}$.

Denote by $\operatorname{Pr}_{1}$ the outage probability of the link from the SS to relay $R_{j}$. Therefore, $\operatorname{Pr}_{1}$ can be given as:

$$
\operatorname{Pr}_{1}=1-\exp \left(-\gamma^{*} \sigma_{0}^{2} / 2 \bar{h}_{\mathrm{B}}^{2} P_{\mathrm{S}}\right) .
$$

Hence the probability that $\Theta_{\mathrm{s}}$ has $n$ relays can be given by:

$$
\operatorname{Pr}_{\mathrm{B}}\left(n ; J, \operatorname{Pr}_{1}\right)=\left(\begin{array}{c}
J \\
n
\end{array}\right)\left(1-\operatorname{Pr}_{1}\right)^{n}\left(\operatorname{Pr}_{1}\right)^{J-n} \text {. }
$$

In the second step of the selection, each SR in $\Theta_{\mathrm{s}}$ decides whether to participate in the forwarding based on the SRIC from the SS together with channel quality of the interference link from itself to the PU. More precisely, a relay $\mathrm{R}_{j} \in \Theta_{\mathrm{s}}$ decides whether to join the subsequent forwarding frame based 


$$
\begin{gathered}
\operatorname{Pr}_{\mathrm{IV}}\left(I_{\mathrm{R}}^{\mathrm{s}}\right)=\sum_{n=1}^{J} \sum_{m=1}^{n}\left(\begin{array}{c}
J \\
n
\end{array}\right)\left(1-\operatorname{Pr}_{1}\right)^{n}\left(\operatorname{Pr}_{1}\right)^{J-n}\left(\begin{array}{c}
n \\
m
\end{array}\right)\left(1-\operatorname{Pr}_{\mathrm{e}}\left(I_{\mathrm{R}}^{\mathrm{s}}\right)\right)^{m}\left(\operatorname{Pr}_{\mathrm{e}}\left(I_{\mathrm{R}}^{\mathrm{s}}\right)\right)^{n-m} \cdot 1_{P_{\mathrm{I}-\mathrm{sum}}}\left(I_{\mathrm{R}}^{\mathrm{s}}\right) . \\
\operatorname{Pr}_{\text {sec,multi }}^{\text {out }}=\sum_{n=1}^{J} \sum_{m=1}^{n}\left(\begin{array}{c}
J \\
n
\end{array}\right)\left(1-\operatorname{Pr}_{1}\right)^{n}\left(\operatorname{Pr}_{1}\right)^{J-n}\left(\begin{array}{c}
n \\
m
\end{array}\right)\left(1-\operatorname{Pr}_{\mathrm{e}}\left(I_{\mathrm{R}}\right)\right)^{m}\left(\operatorname{Pr}_{\mathrm{e}}\left(I_{\mathrm{R}}\right)\right)^{n-m} \cdot \operatorname{Pr}_{2}(m) .
\end{gathered}
$$

on the result of comparing $h_{j, \mathrm{P}}^{2}$ to the SRIC. For instance, if $P_{\mathrm{R}} \cdot h_{j, \mathrm{P}}^{2}>I_{\mathrm{R}}$, then this relay $\mathrm{R}_{j}$ will keep silent in the subsequent relaying frame. The determination of $I_{\mathrm{R}}$ will be discussed in the next subsection.

Based on the topology simplification introduced in Section II, the probability that the interference power of a secondary relay is exceeding the SRIC can be obtained by:

$$
\operatorname{Pr}_{\mathrm{e}}\left(I_{\mathrm{R}}\right)=\exp \left(-I_{\mathrm{R}} / 2 \overline{\mathrm{h}}_{\mathrm{I}}^{2} \mathrm{P}_{\mathrm{R}}\right) .
$$

We denote the final forwarding relay set as $\Theta_{\mathrm{f}}$, so the total interference power to PU can be obtained by:

$$
P_{\mathrm{I}-\mathrm{sum}}=\sum_{R_{j} \in \Theta_{\mathrm{f}}} P_{\mathrm{R}} \cdot h_{j, \mathrm{PU}}^{2} \cdot
$$

If the number of surviving relays is $n$, the distribution of number of relays in the $\Theta_{\mathrm{f}}$, denoted by $m$, can given as:

$$
\operatorname{Pr}_{\mathrm{B}}\left(m ; n, \operatorname{Pr}_{\mathrm{e}}\right)=\left(\begin{array}{l}
n \\
m
\end{array}\right)\left(1-\operatorname{Pr}_{\mathrm{e}}\right)^{m}\left(\operatorname{Pr}_{\mathrm{e}}\right)^{n-m} \text {. }
$$

\section{B. The Value of Single Relay Interference Constraint}

It remains to answer how the SS determines $I_{\mathrm{R}}$. Obviously, the SRIC is strongly dependent on the total interference constraint (denoted by $I_{\text {sum }}$ ) to the PU. In the following we study $I_{\mathrm{R}}$ under two interference constraint policies:

1) Absolute total interference constraint (AIC): This is a common interference constraint in cognitive networks. Under AIC, the sum of interference should be smaller than the threshold $I_{\text {sum }}$ with the probability of 1 . In our distributed relay selection process, the SS doesn't know the exact number of forwarding relays. Therefore, in order to ensure the total interference constraint, the SS should decide the $I_{\mathrm{R}}$ based on the maximum forwarding relay number $J$ which is actually the number of relays deployed in the system. Hence the corresponding SRIC under AIC can be given by:

$$
I_{\mathrm{R}}^{\mathrm{a}}=I_{\mathrm{sum}} / J .
$$

An SR $R_{j}$ keeps silent if $P_{\mathrm{R}} \cdot h_{j, \mathrm{P}}^{2}>I_{\mathrm{R}}^{\mathrm{a}}$. Therefore, the actual sum interference to the PU has the upper bound $I_{\text {sum }}$ :

$$
\sum_{R_{j} \in \Theta_{\mathrm{f}}} P_{\mathrm{R}} \cdot h_{j, \mathrm{P}}^{2} \leq m \cdot I_{\mathrm{R}}^{\mathrm{a}} \leq J \cdot I_{\mathrm{R}}^{\mathrm{a}}=I_{\mathrm{sum}},
$$

where $\Theta_{\mathrm{f}}$ is the forwarding SR set with size $m, m \leq J$.

2) Statistical Total Interference Constraint (SIC): Different from the absolute constraint, the SIC has more accurate requirements from a secondary transmission of interference constraint by introducing a new constraint parameter called interference-violation-probability constraint (IVPC). In other words, with a certain probability constraint SIC allows the interference from the secondary transmission accidentally exceeding the threshold. In practice, the transmission of PU may have certain Quality-of-Service (QoS) requirements, e.g., outage probability or delay. If the IVPC is one magnitude lower than the outage probability requirement of PU, the negative impact from the secondary transmission on the QoS of PU is still negligible.

Denote the SIC as $I_{\mathrm{R}}^{\text {ac }}$, hence the $\operatorname{Pr}_{\text {iv }}\left(I_{\mathrm{R}}^{\mathrm{s}}\right)$, which denotes the interference violation probability of the system on a given SIC, can be expressed by (3). In (3), the $1_{P_{\mathrm{I}-\text { sum }}}\left(I_{\mathrm{R}}^{\mathrm{s}}\right)$ is an indicator function on whether the interference power received by PU $P_{\mathrm{I}-\text { sum }}$ exceeds the threshold $I_{\text {sum }}$ and given by:

$$
1_{P_{\mathrm{I}-\mathrm{sum}}}\left(I_{\mathrm{R}}^{\mathrm{s}}\right)= \begin{cases}1, & \text { if } P_{\mathrm{I}-\operatorname{sum}}\left(I_{\mathrm{R}}^{\mathrm{s}}\right)>I_{\text {sum }} \\ 0, & \text { if } P_{\mathrm{I}-\operatorname{sum}}\left(I_{\mathrm{R}}^{\mathrm{s}}\right) \leqslant I_{\text {sum }} .\end{cases}
$$

Hence, for a given IVPC, denoted by $\operatorname{Pr}^{\mathrm{c}}$, the SS is able to decide the value of $I_{\mathrm{R}}^{\mathrm{s}}$ by solving:

$$
\operatorname{Pr}_{\mathrm{iv}}\left(I_{\mathrm{R}}^{\mathrm{s}}\right)=\operatorname{Pr}^{\mathrm{c}} \text {. }
$$

As we know, the high $I_{\mathrm{R}}^{\mathrm{ac}}$ activates more relays in forwarding frame, and thus, generates more interferences to the PU. As a result, this leads to a high interference violation probability. Therefore, the $\operatorname{Pr}_{\mathrm{IV}}\left(I_{\mathrm{R}}^{\mathrm{s}}\right)$ is increasing in $I_{\mathrm{R}}^{\mathrm{ac}}$. Hence, (11) has a unique solution on $I_{\mathrm{R}}^{\mathrm{s}}$ which can be found numerically by iterative searching.

\section{Outage Probability Analysis}

In a relaying frame, all relays in set $\Theta_{\mathrm{f}}$ are able to forward the packet to the destination. Then, the SNR at the destination is simply the sum of the SNR of the individual links. Therefore, we have $\gamma_{\mathrm{D}}=\sum_{j \in \Theta_{\mathrm{f}}} \gamma_{j, \mathrm{D}}$. Based on our previous work [13], $\gamma_{\mathrm{D}}$ is a gamma distributed variable with the scale parameter $\beta=2 P_{\mathrm{R}} \bar{h}_{\mathrm{R}}^{2} / \sigma^{2}$. The outage probability of the relaying frame, denoted by $\operatorname{Pr}_{2}(m)$, is a cumulative distribution function of the gamma distribution. For a $\Theta_{\mathrm{f}}$ with $m$ relays, the outage probability of relaying frame is:

$$
\operatorname{Pr}_{2}(m)=\left\{\begin{array}{l}
1-\sum_{j=0}^{m-1} \frac{1}{j !}\left(\frac{\gamma^{*}}{\beta}\right)^{j} e^{-\frac{\gamma^{*}}{\beta}} ; m>0 \\
1 ; \quad m=0
\end{array},\right.
$$

where $\gamma^{*}$ is SNR threshold introduced in Section II. Combining (2) and (7) with (12), the expected outage probability of the two frame secondary transmission is obtained by (4) in which $I_{\mathrm{R}}$ is either $I_{\mathrm{R}}^{\mathrm{s}}$ or $I_{\mathrm{R}}^{\mathrm{a}}$.

In particular, if the secondary network only has one relay, the outage probability can be given by:

$$
\operatorname{Pr}_{\text {sec,single }}^{\text {out }}=\left(1-\operatorname{Pr}_{1}\right)\left(1-\operatorname{Pr}_{\mathrm{e}}\left(I_{\mathrm{R}}\right)\right) \text {. }
$$


Obviously, the outage probability of the secondary transmission is increasing in the packet size. The related minimal outage probabilities are achieved as long as the packet size $\rho$ goes to 0 . Therefore, the multi-relay assisted secondary transmission satisfies:

$$
\lim _{\rho \rightarrow 0} \operatorname{Pr}_{1}=\lim _{\gamma^{*} \rightarrow 0}\left\{1-\exp \left(-\gamma^{*} \sigma_{0}^{2} / 2 \bar{h}_{\mathrm{B}}^{2} P_{\mathrm{S}}\right)\right\}=0,
$$

$\lim _{\rho \rightarrow 0} \operatorname{Pr}_{2}(m)=\left\{\begin{array}{l}1-\lim _{\gamma^{*} \rightarrow 0} \sum_{j=0}^{m-1} \frac{1}{j !}\left(\frac{\gamma^{*}}{\beta}\right)^{j} e^{-\frac{\gamma^{*}}{\beta}}=0 ; m>0 \\ 1 ; \quad m=0\end{array}\right.$

Equation (14) shows that all the relays are able to decode the packet successfully. In addition, it is indicated by (15) that the SU is able to decode the packet correctly as long as the final forwarding relay number is not zero. In other words, the minimal outage probability of the secondary transmission is equal to the probability of all the SR-PU interference links violating the single relay interference constraint.

As a result, the minimal outage probability of the studied multi-relay assisted secondary transmission satisfies:

$$
\lim _{\rho \rightarrow 0} \operatorname{Pr}_{\text {sec,multi }}^{\text {out }}=\operatorname{Pr}_{\mathrm{e}}\left(I_{\mathrm{R}}\right)^{J} \text {. }
$$

Hence, we have the following proposition:

Proposition 1. For a CRN where only the average CSI is available at the SS, the minimal outage probability of the secondary transmission is mainly subject to not the secondary transmission links but the interference links.

In particular, the minimal outage probability of multi-relay scheme under the AIC policy is obtained by:

$\lim _{\rho \rightarrow 0} \operatorname{Pr}_{\text {sec,multi }}^{\text {out }}=\operatorname{Pr}_{\mathrm{e}}\left(I_{\text {sum }} / J\right)^{J}=\exp \left(-I_{\text {sum }} / 2 \bar{h}_{\mathrm{I}}^{2} \mathrm{P}_{\mathrm{R}}\right)$.

On the other hand, the minimal outage probability of a single SR assisted transmission under the AIC policy:

$$
\lim _{\rho \rightarrow 0} \operatorname{Pr}_{\text {sec,single }}^{\text {out }}=\operatorname{Pr}_{\mathrm{e}}\left(I_{\text {sum }}\right)=\exp \left(-I_{\text {sum }} / 2 \bar{h}_{\mathrm{I}}^{2} \mathrm{P}_{\mathrm{R}}\right) \text {. }
$$

Obviously, the following relationship holds:

Proposition 2. Under the AIC policy, the single relay and multi-relay assisted secondary transmissions have the same minimal outage probability if the SS only has the average CSI:

$$
\lim _{\rho \rightarrow 0} \operatorname{Pr}_{\text {sec,multi }}^{\text {out }}=\lim _{\rho \rightarrow 0} \operatorname{Pr}_{\text {sec,single }}^{\text {out }}
$$

\section{NUMERICAL RESULTS AND DISCUSSION}

In this section, we evaluate the proposed relay selection scheme and show the appropriateness of our analytical model by simulations. We randomly deploy 10 relays in a circular area with radius $10 \mathrm{~m}$ while the distances of the SS-SRs, SRsSU and SRs-PU links are set as $100 \mathrm{~m}, 100 \mathrm{~m}$ and $300 \mathrm{~m}$. We assume that the center frequency is $2 \mathrm{GHz}$ and the frame length is set to $T_{\mathrm{f}}=10 \mathrm{~ms}$. Besides, we set $P_{\mathrm{S}}=30 \mathrm{dBm}, P_{\mathrm{R}}=20$ $\mathrm{dBm}$ and $\sigma^{2}=-90 \mathrm{dBm}$. For calculating the path-loss, we utilize the well-known COST231 model. In the simulations, we obtain the outage probabilities of the multi-relay CRN by varying the packet sizes and system topologies.

First, the theoretical and simulation values of the outage probabilities of the secondary transmission versus packet sizes are shown in Fig. 2. The theoretical values are calculated by the above equations while the simulation values are obtained statistically based on randomly generated channels. As a result, Fig. 2 shows the appropriateness of our analytical model. The theoretical values nicely match the simulation values while slight mismatch is caused by the topology simplification.

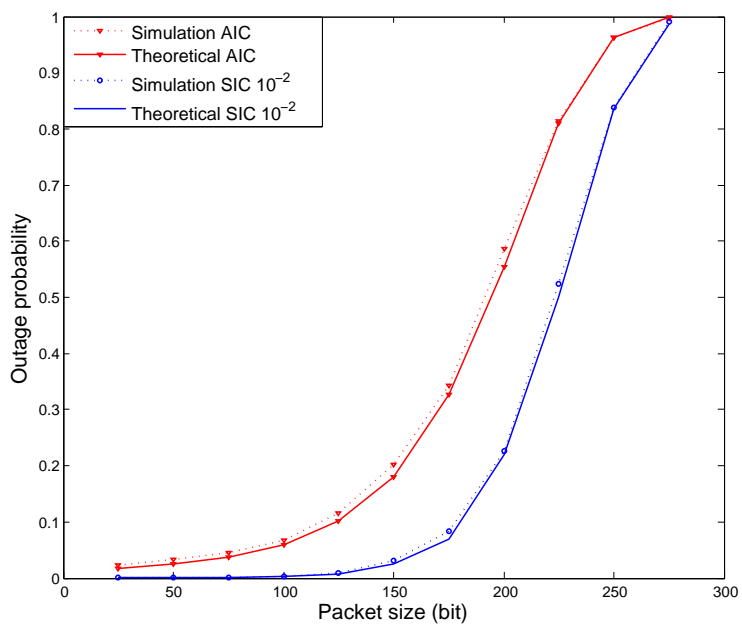

Fig. 2. The theoretical and simulation values of the outage probability of the secondary transmission.

Furthermore, we show the outage probability performances under different interference constraint policies and compare the multi-relay transmission to single relay schemes in Fig. 3. As references, the single relay schemes have the same total interference constraints as the multi-relay schemes. Therefore, the single relay schemes have significantly loose (high threshold) interference constraints for (each) relay in comparison to multi-relay transmission. We learn the following intriguing re-

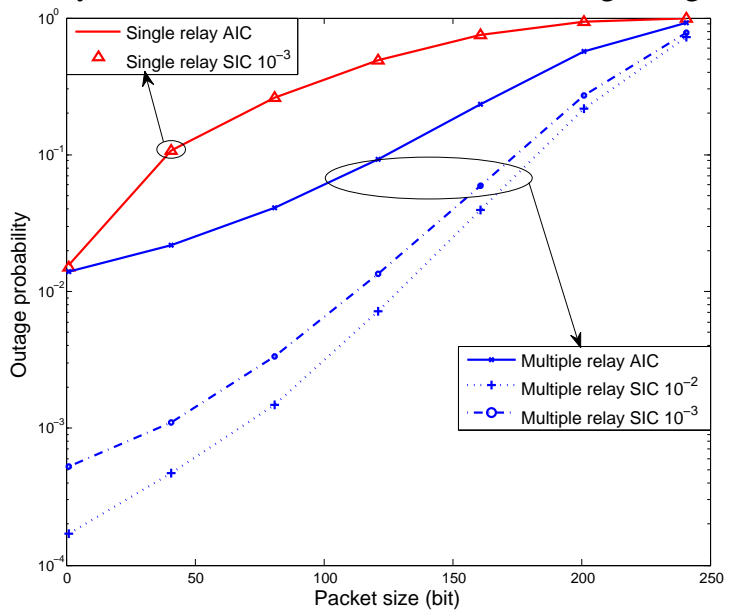

Fig. 3. The outage probability of the secondary transmission under different interference constraints.

lationships among the curves. Firstly, under AIC policy, multirelay and single relay assisted secondary transmissions almost have the same outage performance with extreme short packets. 
This matches our analysis that the above two transmissions have the same value of the minimal outage probabilities under the AIC policy. Secondly, compared to the single relay CRN, although having a lower interference constraint at each relay, multi-relay CRN performances lie in the optimal positions (for nonzero packet size). Therefore, for a multi-relay CRN where the SS only has the average channel gains information, an appropriate policy of guaranteeing the primary transmission does not let only one SR assist the secondary transmission but keeps utilizing the multi-relay and gives each SR a relatively lower/stringent interference constraint. Thirdly, the SIC schemes are more reliable than the AIC scheme for multirelay transmissions. Even if the IVPC is as low as $10^{-3}$, the SIC is still significantly superior to AIC. At the same time, SIC and AIC have the same performances when the secondary network only has one relay. In other words, SIC is only promising in multi-relay CRN.

Another comparison between the single-relay and multirelay schemes is shown in Fig. 4 by varying the SRs-PU distance from $300 \mathrm{~m}$ to $100 \mathrm{~m}$ and SRs-SU distance from $100 \mathrm{~m}$ to $300 \mathrm{~m}$. When the PU is close to the SR group,

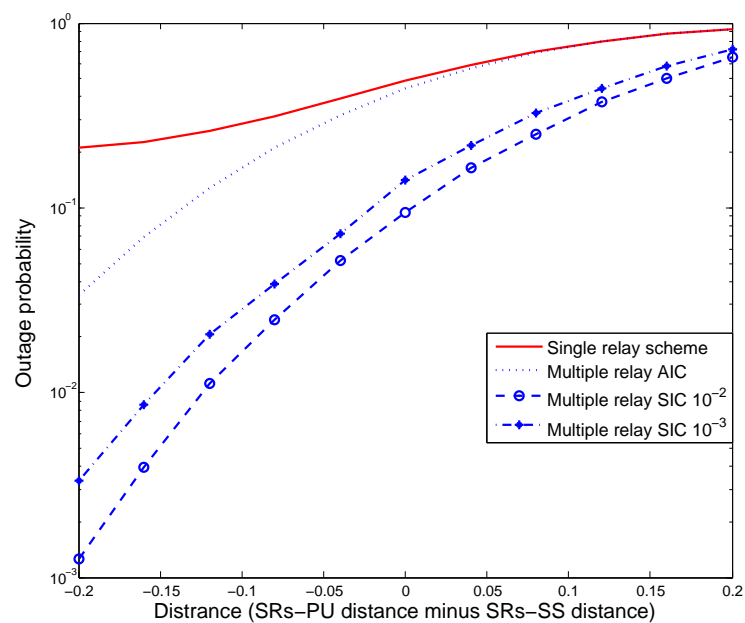

Fig. 4. The outage probability of the secondary transmission while the system topology is varied. The Packet size is set as 100 bits.

the SRs-PU interference channels become strong. In order to protect the primary transmission, the secondary transmission is severely limited, i.e., relays cannot forward the packet to the SU with a high probability. On one hand, this limitation makes the outage probability increase as the distance gap (SRs-PU distance minus SRs-SS distance) increases. On the other hand, the flexibility of the multi-relay schemes are weakened by increasing the gap. As a result, the curves of the single relay scheme and the multi-relay AIC scheme converge when the PU is much closer to SRs than to the SU. At the same time, multirelay SIC schemes are still significantly superior to the multirelay AIC scheme. Hence, it is necessary to spend the general overhead for a secondary multi-relay network on knowing the exact QoS requirement of the PU (i.e. the interference violation probability) for applying the SIC policy.

\section{CONCLUSion}

In this paper, we focus on a multi-relay CRN system where the SS only has the knowledge of the average CSI. A simple distributed multi-relay selection scheme is proposed while two interference constraint policies (AIC and SIC) are considered for the selection. The proposed selection scheme indeed protects the PU while providing nice performances for the secondary transmissions. Moreover, as the constraint from the PU gets stringent, in comparison to AIC policy, SIC policy improves the system performance drastically by exploiting the spatial diversity of the multiple relays. This is interesting and different from the traditional relaying. Therefore, our work suggests to spend the general overhead for secondary networks on knowing the exact QoS requirement of the PU. In addition, we analyze the outage probability as well as the minimal outage probability of the multi-relay CRN. We find that the minimal outage probability of a CRN is only subject to the interference links and that the single and multiple relays assisted secondary transmissions have the same minimal outage probabilities under the AIC policy. At last, we show that although having uncertain number of forwarding relays and uncertain interference to the PU, the proposed relay selection scheme let the performance of multi-relay secondary transmissions be absolutely superior to the single-relay.

\section{REFERENCES}

[1] A. Goldsmith, S. Jafar, I. Maric, and S. Srinivasa, "Breaking spectrum gridlock with cognitive radios: An information theoretic perspective," Proc. IEEE, vol. 97, no. 5, May 2009, pp. 894-914.

[2] Y. Kim and H. Liu, "Infrastructure relay transmission with cooperative mimo," IEEE Trans. on Vehicular Technology, , vol. 57, no. 4, Jul. 2008 , pp. $2180-2188$.

[3] A. Adinoyi and H. Yanikomeroglu, "Cooperative relaying in multiantenna fixed relay networks," IEEE Trans. on Wireless Comm., vol. 6, no. 2, Feb. 2007, pp. $533-544$.

[4] A. Bletsas, H. Shin, and M. Win, "Cooperative communications with outage-optimal opportunistic relaying," IEEE Trans. on Wireless Comm., vol. 6, no. 9, Sep. 2007 , pp. $3450-3460$.

[5] Y. Hu and L. Qiu, "A novel multiple relay selection strategy for lteadvanced relay systems," in Proc. IEEE VTC, Budapest,Hungary, May 2011, pp. 1-5.

[6] H. Suraweera, P. Smith, and N. Surobhi, "Exact outage probability of cooperative diversity with opportunistic spectrum access," in Proc. IEEE ICC Comm. Workshop, Beijing, China, May 2008, pp. 79-84.

[7] S. Kim, W. Choi, Y. Choi, J. Lee, Y. Han, and I. Lee, "Downlink performance analysis of cognitive radio based cellular relay networks," in Proc Cognitive Radio Oriented Wireless Networks and Comm.,Singapore, May 2008, pp. 1-6.

[8] X. Zhang, Z. Yan, Y. Gao, and W. Wang, "On the study of outage performance for cognitive relay networks with the nth best-relay selection in rayleigh-fading channels," IEEE Wireless Comm. Letters, vol. 2, no. 1, 2013, pp. 110-113.

[9] D. Chen, H. Ji, and X. Li, "Optimal distributed relay selection in underlay cognitive radio networks: An energy-efficient design approach," in Proc. IEEE WCNC, Cancun, Mexico, Mar. 2011, pp. 1203-1207.

[10] J. Lee, H. Wang, J. Andrews, and D. Hong, "Outage probability of cognitive relay networks with interference constraints," IEEE Trans. on Wireless Comm., vol. 10, no. 2, Feb. 2011, pp. 390-395.

[11] G. Chen, Z. Tian, et al, "Decode-and-forward buffer-aided relay selection in cognitive relay networks," IEEE Trans. on Vehicular Technology, , vol. PP, no. 99, 2014. pp. 1-6.

[12] K. Lee, A. Yener, "Outage performance of cognitive wireless relay networks," Proc. IEEE GLOBECOM, San Francisco, Nov. 2006, pp. 1-5.

[13] Y. Hu and J. Gross, "On the outage probability and effective capacity of multiple decode-and-forward relay system," in Proc. IFIP Wireless Days conference, Dublin, Ireland, Nov. 2012, pp. 1-8. 